\title{
Effect of Home-based-Nursing Intervention on Knowledge, Daily Living Activities and pain for Patients after Coronary Artery Bypass Graft
}

\author{
Nabila E. Saboula ${ }^{1}$, Amal A. Hussein ${ }^{2}$, Abeer G. A. Habouh ${ }^{3}$ \\ Prof. of Community Health Nursing, Faculty of Nursing/ Menoufia University, Egypt- \\ Taif University, $\mathrm{KSA}^{1}$ \\ Ass. Prof. of Community Health Nursing, Faculty of Nursing/ Menoufia University²,
}

Nurse Specialist at Mahalla Cardiac Center, Al-Gharbia Governorate, M.O.H -Egypt 3

\begin{abstract}
:
Background: The advantages of nursing support for cardiac rehabilitation patients can improve the health outcomes and reduce the risk of a new cardiac event through education, support, supervision, and reinforcement .The aim of this study was to assess the effectiveness of home-based-nursing intervention on knowledge, daily living activities and pain for patients after coronary artery bypass graft. Study Design: A quasi experimental research design was utilized. Study Setting: The study was conducted in the out- patient clinics of Mahalla Cardiac Center, Mahalla city, Al-Gharbia Governorate, Egypt. Study Sample: A purposive sample of 140 post- coronary artery graft surgery patients was included. The total sample was divided randomly into two groups study group (70 patients) and control group (70 patients). Study Tools: 1) A structured interviewing questionnaire including; 1 ) the socio- demographic data of studied patients. 2) Medical data including patient information about present and past history .3) Knowledge assessment data. 4) Barthel Index Scale of measuring activities of daily living (BIS). Study Results: After implementing the Home Based Nursing Intervention, there was high statistical significance improvement in study groups' total knowledge compared to controls. Pain level had significantly improved, as well as daily living activities among study group than control group. Conclusion: Home based nursing intervention was effective in improving patient's knowledge, level of pain and daily living activities after coronary artery bypass graft. Also, it played an important role in improving those patients through tailoring health education about healthy life styles for complete recovery and preventing complications. Recommendations: Nurses have to povide awareness program for educating patients about Home based nursing intervention to prevent complication and attaining complete recovery after coronary artery bypass graft (CABG) surgery.
\end{abstract}

Keywords: Home Based Nursing Intervention, Coronary Artery by pass Graft, Knowledge, Daily Living Activities and Pain.

Vol. 18 No. 1 May, 2020 


\section{INTRODUCTION}

Coronary artery disease (CAD) is the most common form of heart diseases. It is estimated that nearly one half of all middle-aged men and one third of middleaged women in the United States will develop some form of the disease. CAD is the number one killer in the developed world, with over 7.4 million deaths attributed to CAD in $2012^{(1) .}$

According to the latest world health organization data published in 2017, coronary artery disease is as a leading cause of death and disability in all around the world. Coronary heart disease death in Egypt accounts for126, 312 or $24.58 \%$ of total deaths. The age adjusted death rate is 216.82 per 100.000 of population. Egypt ranks number18 in the world (WHO, 2017) (2). Since the mean prevalence of CAD is estimated to be $6.9 \%$ in men and $6.0 \%$ in women, it is important for nurses to become familiar with the various types of coronary artery conditions and the methods for assessing, preventing, and managing these disorders medically and surgically (Direk and Şenol Çelik , 2012) ${ }^{\text {(3) }}$

Worldwide, each year more than 300,000 patients is undergo (CAPG) surgery. Approximately one-fifth of them will have recurrence of coronary heart disease symptoms within the first 5 years. This risk increases with age and is higher among women. Up to $30 \%$ will have angina in the first postoperative year, increased risk for myocardial infarction, and greater need for re-operation (4)

The prevalence of risk factors for cardiovascular disease was relatively high among both Saudi and Egyptian medical students, particularly a sedentary life style, obesity, and abdominal obesity. Smoking was practiced by $29.7 \%$ of both populations. A significantly higher prevalence of obesity and a reported family history of premature coronary artery disease (CHD) were observed among the Saudi students and a significantly higher prevalence of hypertension was found among male Egyptian students as compared with male Saudi students. A relatively high proportion of both populations $(23.9 \%$ of Saudi students and $16.7 \%$ of the Egyptian students) was at an increased risk of developing fatal cardiovascular disease within 10 years (Heron ,2011) ${ }^{(5)}$

CABG is indicated for patients with more than two arterial constrictions, with weakened left ventricles, or with diabetes. There are other therapies for patients 
whose medical treatment does not improve the symptoms of their coronary artery disease but who are not good candidates for either PCI or CABG. The alternatives include laser trans myocardial revascularization (using a carbon dioxide laser), enhanced external counter pulsation to reduce the frequency of angina, and spinal cord stimulation to relieve the pain of angina (Bhimji, 2013 and McLaughlin, 2014). ${ }^{(6,7)}$

A sedentary lifestyle is a risk factor for CAD. Patients with a sedentary lifestyle are also more likely to also be overweight or obese, which contributes to the risk developing CAD. Patient goals for physical activity should begin with 10 to 15 minutes a day and gradually work up to a goal of 30 minutes a day of moderate to vigorous exercise. The more vigorous the activity, the greater the benefits. The level of activity should be based on the patient's baseline condition and other comorbid diseases. Patients should always work with their healthcare provider prior to starting an exercise program ${ }^{(7)}$

Home based nursing guidelines after coronary artery by bass graft surgery promotes cardiac rehabilitation and that are focused on risk factor modifications, adequate exercise training, and coping strategies are effective measures to reduce recurrent events. For most patients, heart surgery is a life-saving procedure but also a major source of emotional stress and financial burden (Heran, 2011). [8].Knowledge is an important method for all patients with cardiac diseases, to improve his health status and prevent of complication and classified into actual knowledge positive or definite, personal knowledge based on one's own observation, and constructive knowledge based on other circumstances (Temple, $2010^{\text {(9). }}$

The Activities of Daily Living (ADLs) are defined as" set of activities necessary for normal self-care. For those patients with cardiac disease .The activities include movement in bed, transfers, locomotion, dressing, personal hygiene, and feeding pattern. Health care providers are under constant pressure to discharge patients quickly, but it is essential that nurses engage early in adequate, individualized, and in-depth discharge planning, that increasing the odds for successful recovery ${ }^{(10)}$

Cardiac rehabilitation is continuing to evolve to meet a variety of age groups and needs. Advanced age is associated with a higher prevalence of CAD as well as 
increased morbidity and mortality. Cardiac rehabilitation programs designed to meet the needs of older patients ( $>65$ years of age) should include strength, balance, coordination, and flexibility. Evidencebased programs show that elderly patients can realize positive benefits from an exercise-based cardiac rehabilitation program to increase functional capacity, glucose control, quality of life, enhanced ability to perform ADLs, and reduced incidence of hospitalization (Menezes et al., 2014). ${ }^{(11)}$

\section{AIM OF THE STUDY}

The aim of this study was to assess the effectiveness of home -based-nursing intervention on knowledge, daily living activities and pain for patients after coronary artery bypass graft surgery.

\section{RESEARCH HYPOTHESES:}

1. Patients who receive home based nursing intervention after coronary artery bypass graft will have improved knowledge score compared to control group.

2. Patients who receive home based nursing intervention after coronary artery bypass will have better daily living activities score compared to control group.

3. Patients who receive home based nursing intervention after coronary artery bypass will have lower pain score compared to control group.

\section{Material and methods}

Design :- A quasi experimental research design (case\& control was utilized.

Setting :- This study was conducted in the out- patient clinics of Mahalla Cardiac Center, Mahalla city, Al-Gharbia Governorate, Egypt.

Subjects

- A purposive sample of 140 postcoronary artery graft surgery patients was recruited. The total sample size was divided into two groups according to age, and education. A study group composed of 70 patients who received home based nursing intervention (HBNI) while control group composed of 70 patients who received the routine follow up care.

\section{Inclusion criteria:}

- Patient immediately discharged from the hospital after coronary artery bypass grafting (CABG).

- Both sex

- Age more than 30 years.

\section{Exclusion criteria:}

-Patient with other cardiac surgery such as valve replacement or congenital heart diseases. 


\section{Sample Size:}

The following power analysis equation was used to indicate the study sample size. Prior data indicated that the prevalence of knowledge among control group is $30 \%$. If the true knowledge rate for intervention subjects is $60 \%$, we will need to study 70 experimental subjects and 70 control subject to be able to reject the null hypothesis that the knowledge rate for experimental and control subjects are equal with probability power 0.9 . The Type 1 error probability associated with this test of this null hypothesis is 0.05 . The researcher used an uncorrected chi- squared statistic to evaluate this null hypothesis.

\section{Tools}

Data was collected through using the following tools:

1- A structured interviewing questionnaire developed by the researcher depending on a review of related literature, which include the following:

A- Socio- demographic data which included name, age, sex, marital status occupation, address, level of education and monthly income....

B-Medical data which included patient information about present and past history as frequency of hospital admission and regular follow up of surgery from patients/ or ICU report.

C-Knowledge assessment: which included information about nature of the operation, treatment regimen, warning signs after operation, complications.

\section{Scoring system of knowledge:}

The questionnaire contained items related to CABG patients' demographic criteria, as well as four patients' knowledge assessment subscales each was two points Liker Scale $(0-2)$ as $(0)$ for wrong answer and (I) correct but not complete, and (2) for correct and complete answer.

The knowledge about factors that lead to CAD, as well as post-operative complications was evaluated giving a score of 0-12. The total score of each patient was categorized into "poor knowledge" when he/she achieved less than or equal $\leq 50 \%$ of the total score, and "good knowledge" was considered when the patient achieved more than $50 \%$ of the total score. Accordingly, patients who had from 0-6 points of the total score, were considered as having "poor knowledge", and those who had 7-12 points were considered as having "good knowledge".

The knowledge total score was evaluated giving a score of 0-44. The total score of each patient was categorized into "poor

Vol. 18 No. 1 May, 2020 
knowledge" when he/she achieved less than or equal $\leq 50 \%$ of the total score, and "good knowledge" was considered when the patient achieved more than $50 \%$ of the total score. Accordingly, patients who had from 0-22 points of the total score, were considered as having "poor knowledge", and those who had 23 - 44 points were considered as having" good knowledge".

2- Barthel Index Scale for measuring Activities of Daily Living (BIS) that was developed by Barthel Collin et al., (1988) to measures a person's daily functioning specifically the activities of daily living and mobility ${ }^{(12) .}$

3-Visual analogue Scale (VAS) that was developed by Wewers \& Lowe, (1990) to measure the intensity of pain ${ }^{(13) .}$

\section{Reliability of the tools:}

Test-retest reliability was applied using a group of 10 patients who were not included in the study. The tool was presented to them twice- two weeks apart. The tool proved to be strongly reliable $(\mathrm{r}=$ 0.8222). When used to measure improvement after the HBNI, changes of more than two points in the total score reflect a probable genuine change, and change on one item from fully dependent to independent is also likely to be reliable.

Validity of the tools:
The scale was tested for its content validity by group of five experts in the community nursing, medical surgical medicine and nursing and psychiatric nursing, to ascertain relevance and completeness. The relevancy, clarity, fluency, and simplicity of each component in the questionnaire were examined by the expert.

\section{Pilot Study:}

A pilot study was carried out on 10 subjects to assess the clarity, feasibility, applicability of the study tools, and time needed to fill the tool. The necessary modification was done as revealed from the pilot study. The sample of pilot study was excluded from the total sample to assure the stability of the results.

\section{Ethical Consideration:}

For ethical reasons the protocol was approved by the ethical \& scientific research committee of the "Faculty of Nursing, Menoufia University”. An official permission was taken from the directors of out- patient clinic. Before data collection, each subjects was informed about the aim of the study and its importance. They were given an opportunity to refuse to participate. Also they were assured that the information would remain confidential and used for the research purpose only. 


\section{Methods}

- Duration of study: data was collected at the beginning of September, 2017 to the end of Feb, 2018.

- An interview was done with the participants at the outpatient clinic to explain the purpose of the study and its importance to guarantee their cooperation and commitment.

- The study intervention's components runs according to the American Heart Association, (2016).

- A base line assessment was done for all participants immediately at discharging from the hospital and then study group were followed at home by phone, and control group were followed using their scheduled follow up hospital's list.

-Participants in the study group received the (HBNI) at the out-patient clinic of Mahala Cardiac Center, and followed up through their homes, while the control group received the routine hospital care and guidelines. The study group was divided to groups of 5 each to receive the designed educational session conducted at the outpatient clinic at their scheduled appointments with their doctor. Then they were followed by telephone if they miss a follow up schedule with their surgeon's for confirmation of commitment to the study and the healthy content of the sessions.

- The intervention included five educational sessions and awareness about medication regimen, therapeutic diet, sleep hygiene, walking, physical activity, pain management.

-The intervention started immediately with the study group after discharge from the outpatient Cardiac Center clinic and continued for the first five weeks.

- Assessment of knowledge, daily living activities and level of pain and its characteristics for studied patients were done, then compere (After one month from the intervention) the study group with control group. Assessment of pain using VAS scale to measure pain intensity and assessment of ADLs was done using the BIS scale.

-Interventional sessions were conducted in the form of presentation educational sessions contents using data show in the out- patient department. It is a highly structured method by which the community nurse verbally and visually transmits information directly to the study group of coronary artery bypass graft.

\section{Statistical analysis:}


Data was coded and transformed into specially designed form to be suitable for computer entry process. Data was entered and analyzed by using SPSS (Statistical Package for Social Science) statistical package version 22. Graphics were done using Excel program. Quantitative data were presented by mean (X) and standard deviation (SD). It was analyzed using student t- test for comparison between two means .Qualitative data were presented in the form of frequency distribution tables, number and percentage. It was analyzed by chi-square $\left(\chi^{2}\right)$ test.

\section{RESULTS}

Table (1) it revealed that there was no statistical significance differences between study group and control in all sociodemographic characteristics of the studied patients at the beginning of the study.

Table (2) it clarified that there was no statistical significance differences between study group and control in all medical and family history.

Table (3) it highlighted a high statistical significant effect of home based nursing intervention on the four knowledge aspects among the study group compared to control group. The table revealed a high statistical significant improvement $(\mathrm{p}<$ 0.000) in the different subscales of knowledge.
Figure (1) it showed the effect of the home based nursing intervention on patient's knowledge. The graph clarified that almost three fourth percent $(74.3 \%)$ of the study group of patients had good knowledge score compared to $37.1 \%$ of the control group.

Table (4) it highlighted the efficacy of home -based-nursing intervention on the four knowledge subscales as well as the total knowledge scores among study and control patients groups. The table revealed a highly significant improvement $(\mathrm{p}<0.000)$ in the different aspects of knowledge aspects. and $74.3 \%$ for total knowledge. While The good knowledge responses among control group ranged from $17.1 \%$ for wound \& skin care to $48.6 \%$ for post-operative complications

Figure (2) it clarified that only $1.6 \%$ of the study group became moderately independent after the home-based nursing intervention on total score of daily living activities compared to $88.6 \%$ e of the control group.

Table (5) it revealed that there was high statistical significance decrease on pain among the study group than the control group after implementing the home based nursing intervention. 
Table (1): Distribution of the studied patients after coronary artery bypass operation according to the socio -demographic characteristics $(N=140)$

\begin{tabular}{|c|c|c|c|c|c|c|}
\hline \multirow{3}{*}{$\begin{array}{l}\text { Socio -demographic } \\
\text { characteristics }\end{array}$} & \multicolumn{4}{|c|}{ Groups } & \multirow[t]{3}{*}{ Test of Sig. } & \multirow{3}{*}{ P-value } \\
\hline & \multicolumn{2}{|c|}{ Study Group(70) } & \multicolumn{2}{|c|}{ Control Group (70) } & & \\
\hline & No. & $\%$ & No. & $\%$ & & \\
\hline \multicolumn{7}{|l|}{ Age groups } \\
\hline $30-50$ Years & 44 & $62.9 \%$ & 36 & $51.4 \%$ & \multirow{2}{*}{$X^{2}=0.9$} & \multirow{2}{*}{$\mathrm{P}=0.3 \mathrm{NS}$} \\
\hline $51-70$ Years & 26 & $37.1 \%$ & 34 & $48.6 \%$ & & \\
\hline $\mathrm{X} \pm$ SD & \multicolumn{2}{|c|}{$43.7 \pm 4.3$} & \multicolumn{2}{|c|}{$45.6 \pm 8.4$} & $\mathrm{t}=1.1$ & $\mathrm{P}=0.8 \mathrm{NS}$ \\
\hline \multicolumn{6}{|l|}{ Sex } & \multirow{3}{*}{$\mathrm{P}=0.73 \mathrm{NS}$} \\
\hline Male & 37 & $52.8 \%$ & 39 & $55.7 \%$ & \multirow{2}{*}{$X^{2}=0.12$} & \\
\hline Female & 33 & $47.2 \%$ & 31 & $44.3 \%$ & & \\
\hline \multicolumn{5}{|l|}{ Marital status } & \multirow{3}{*}{$\mathrm{X} 2=0.0$} & \multirow[t]{3}{*}{$\mathrm{P}=1.0 \mathrm{NS}$} \\
\hline Married & 34 & $48.6 \%$ & 34 & $48.6 \%$ & & \\
\hline Unmarried & 36 & $51.4 \%$ & 36 & $51.4 \%$ & & \\
\hline \multicolumn{6}{|l|}{ Education } & \multirow{4}{*}{$\mathrm{P}=0.73 \mathrm{NS}$} \\
\hline Illiterate & 16 & 22.9 & 20 & 28.6 & \multirow{3}{*}{$\mathrm{X}^{2}=0.11$} & \\
\hline $\begin{array}{l}\text { Elementary \& intermediate } \\
\text { education }\end{array}$ & 32 & 45.7 & 28 & 40 & & \\
\hline University & 22 & 31.4 & 22 & 31.4 & & \\
\hline \multicolumn{5}{|l|}{ Occupation } & \multirow{5}{*}{$\mathrm{X}^{2}=1.3$} & \multirow{5}{*}{$\mathrm{P}=0.4 \mathrm{NS}$} \\
\hline Farmer, landowner\& pension. & 20 & 28.6 & 14 & 20 & & \\
\hline Housewife & 6 & 8.6 & 16 & 22.4 & & \\
\hline Employee \&worker & 6 & 8.6 & 20 & 28.6 & & \\
\hline Free business & 38 & 54.2 & 20 & 28.6 & & \\
\hline Family income & & & & & & \\
\hline
\end{tabular}

Vol. 18 No. 1 May, 2020 
Tanta Scientific Nursing Journal

\begin{tabular}{|c|c|c|c|c|c|c|}
\hline Salary-pension & 20 & 28.6 & 25 & 42.9 & \multirow{3}{*}{$X^{2}=1.2$} & \multirow{3}{*}{$\mathrm{P}=0.51 \mathrm{NS}$} \\
\hline Agriculural income & 18 & 27.3 & 36 & 51.4 & & \\
\hline Relatives help \& others & 32 & 45.7 & 4 & 5.7 & & \\
\hline \multicolumn{5}{|l|}{ Residence } & \multirow{3}{*}{$X^{2}=0.3$} & \multirow{3}{*}{$\mathrm{P}=0.6 \mathrm{NS}$} \\
\hline Urban & 48 & 68.6 & 44 & 62.9 & & \\
\hline$\overline{\text { Rural }}$ & 22 & 31.4 & 26 & 37.1 & & \\
\hline Total & 70 & 100 & 70 & 100 & & \\
\hline
\end{tabular}

Table 2: Medical and family history of coronary heart disease (study group\& control $(\mathrm{N}=140)$

\begin{tabular}{|c|c|c|c|c|}
\hline \multirow{3}{*}{$\begin{array}{l}\text { Medical and family } \\
\text { history Variables }\end{array}$} & \multicolumn{2}{|c|}{ Groups } & \multirow[t]{3}{*}{ Test of Sig. } & \multirow{3}{*}{ P-value } \\
\hline & Study Group & Control & & \\
\hline & No. & No. & & \\
\hline \multicolumn{4}{|l|}{ Number of hospital stay : } & \\
\hline $1-2$ & 68.6 & 42 & \multirow{2}{*}{$X^{2}=0.61$} & \multirow{2}{*}{$\mathrm{P}=0.4 \mathrm{NS}$} \\
\hline $3-4$ & 31.4 & 28 & & \\
\hline \multicolumn{4}{|c|}{ Number of follow up per month after open heart surgery : } & \multirow{3}{*}{$P=1.0$ NS. } \\
\hline $1-2$ & $62 \quad 88.6$ & 88.6 & \multirow{2}{*}{$X^{2}=0.0$} & \\
\hline $3-4$ & $\begin{array}{ll}8 & 11.4\end{array}$ & 11.4 & & \\
\hline \multicolumn{3}{|c|}{ Family history of coronary heart diseases } & \multirow{3}{*}{$X 2=2.4$} & \multirow{3}{*}{$P=0.12$ NS } \\
\hline Yes & 22.8 & 28 & & \\
\hline No & $54 \quad 77.2$ & 42 & & \\
\hline Total & $\begin{array}{ll}70 & 100 \%\end{array}$ & $\begin{array}{ll}70 & 100 \%\end{array}$ & & \\
\hline
\end{tabular}




\section{Tanta Scientific Nursing Journal}

Table 3 : Study group and control responses to knowledge aspects, and total knowledge assessment questionnaire $(\mathrm{N}=140)$.

\begin{tabular}{|c|c|c|c|c|c|c|c|c|}
\hline \multirow[t]{2}{*}{ Knowledge aspects } & \multicolumn{3}{|c|}{ Study group } & \multicolumn{3}{|c|}{ Control } & \multirow{2}{*}{$\begin{array}{c}\text { Test of } \\
\text { sig. }\end{array}$} & \multirow[t]{2}{*}{ P-value } \\
\hline & $\begin{array}{c}\text { Poor } \\
\text { Knowledge } \\
\text { No. }(\%)\end{array}$ & $\begin{array}{c}\text { Good } \\
\text { knowledge } \\
\text { No. }(\%)\end{array}$ & $\begin{array}{c}\text { Mean } \\
\text { score } \pm \\
\text { SD }\end{array}$ & $\begin{array}{c}\text { Poor } \\
\text { Knowledge } \\
\text { No. }(\%)\end{array}$ & $\begin{array}{c}\text { Good } \\
\text { knowledge } \\
\text { N0. }(\%)\end{array}$ & $\begin{array}{c}\text { Mean } \\
\text { score } \pm \\
\text { SD }\end{array}$ & & \\
\hline CABG nature & $11.4 \%$ & $\begin{array}{ll}69 & 98.6\end{array}$ & $7.2 \pm 1.2$ & $\begin{array}{ll}42 & 60 \%\end{array}$ & $\begin{array}{ll}28 \quad 40 \% \\
\end{array}$ & $4.0 \pm 1.5$ & $\begin{array}{l}X^{2}=31.7 \\
t=10.4\end{array}$ & $\begin{array}{l}\text { 0.000 } \\
\text { HS }\end{array}$ \\
\hline $\begin{array}{l}\text { Factors lead to } \\
\text { CAD }\end{array}$ & $2 \quad 2.9 \%$ & $68 \quad 97.1 \%$ & $8.2 \pm 1.3$ & $\begin{array}{|ll|}40 & 57.1 \\
\end{array}$ & $30 \quad 42.9 \%$ & $6.1 \pm 0.9$ & $\begin{array}{c}X^{2}=48.7 \\
t=8.5\end{array}$ & $\begin{array}{l}\text { 0.000HS } \\
\text { 0.000HS }\end{array}$ \\
\hline $\begin{array}{l}\text { Wound \& skin } \\
\text { care after surgery }\end{array}$ & $4 \quad 5.7 \%$ & $\begin{array}{ll}66 & 94.3 \\
\end{array}$ & $6.9 \pm 1.4$ & $\begin{array}{ll}58 & 82.9 \% \\
\end{array}$ & $12 \quad 17.1 \%$ & $4.8 \pm 1.3$ & $\begin{array}{c}X^{2}=112.5 \\
t=14.6\end{array}$ & $\begin{array}{l}\text { 0.000HS } \\
\text { 0.000HS }\end{array}$ \\
\hline $\begin{array}{l}\text { post- operative } \\
\text { complications }\end{array}$ & $12 \quad 17.1 \%$ & $\begin{array}{ll}58 & 82.9 \%\end{array}$ & $8.6 \pm 2.5$ & $\begin{array}{|ll|}36 & 51.4 \% \\
\end{array}$ & $34 \quad 48.6 \%$ & $5.2 \pm 1.2$ & $\begin{aligned} \mathrm{X}^{2} & =53.2 \\
\mathrm{t} & =9.5\end{aligned}$ & $\begin{array}{l}\text { 0.000HS } \\
0.000 \mathrm{HS}\end{array}$ \\
\hline Total knowledge & $\begin{array}{ll}18 & 25.7 \%\end{array}$ & $\begin{array}{|ll|}52 & 74.3 \% \\
\end{array}$ & $33.2 \pm 2.6$ & $\begin{array}{|ll|}44 & 62.9 \% \\
\end{array}$ & $26 \quad 37.1 \%$ & $28.5 \pm 2.7$ & $\begin{array}{c}X^{2}=19.6 \\
t=6.6\end{array}$ & $\begin{array}{l}\text { 0.000HS } \\
\text { 0.000HS }\end{array}$ \\
\hline
\end{tabular}


Figure 1: Effect of home-based nursing intervention on total score knowledge groups of studied patients

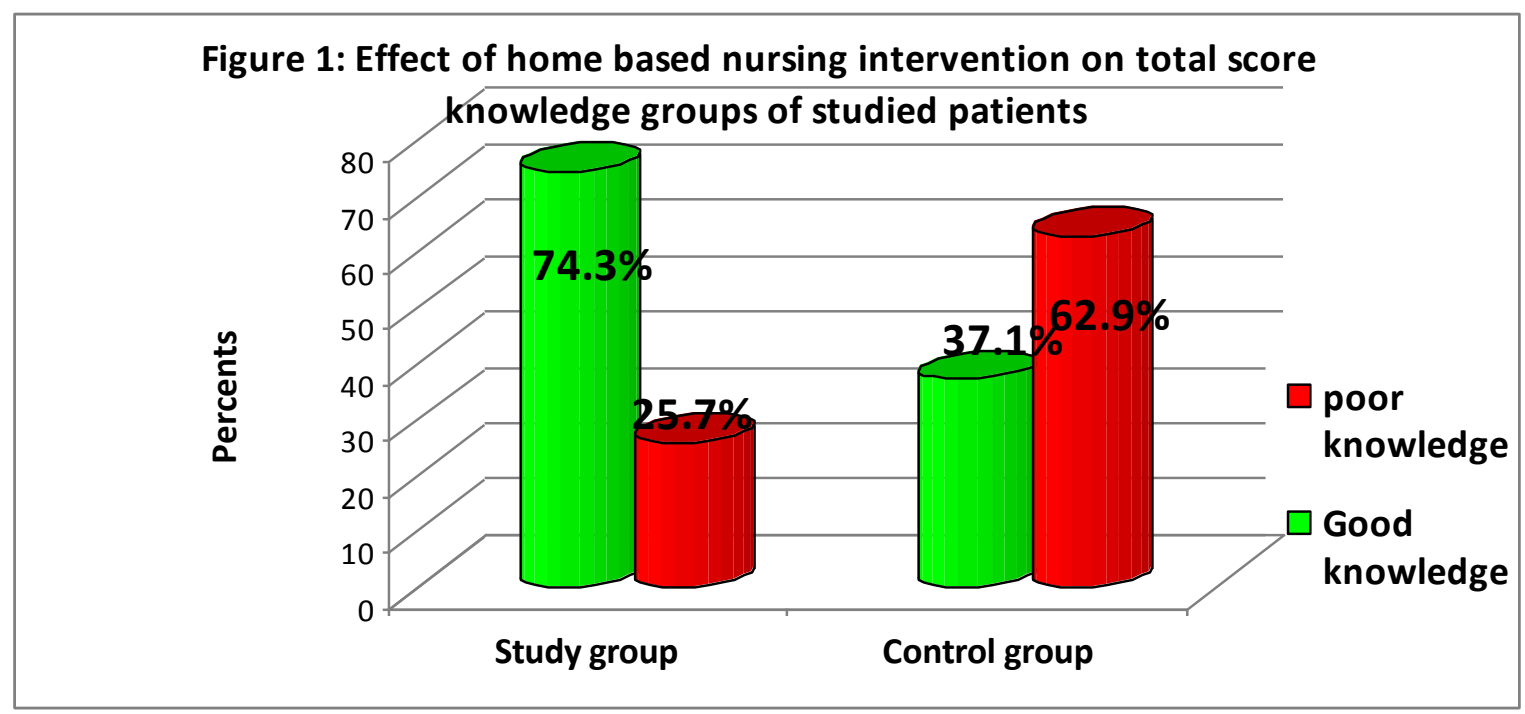

Table (4): Distribution of the studied patients groups according to their Barthel index scale (BIS) to measure activities of daily living $(\mathbf{N}=140)$

Vol. 18 No. 1 May, 2020 


\begin{tabular}{|c|c|c|c|c|c|c|c|}
\hline \multirow[t]{2}{*}{ The patients 'LDAs } & \multicolumn{2}{|c|}{$\begin{array}{c}\text { Completely } \\
\text { dependent (0) }\end{array}$} & \multicolumn{2}{|c|}{ Need help (5) } & \multicolumn{2}{|c|}{$\begin{array}{c}\text { Completely } \\
\text { Independent (10) } \\
\end{array}$} & \multirow{2}{*}{$\begin{array}{l}\text { Test of sig. } \\
P \text { value }\end{array}$} \\
\hline & No & $\%$ & No & $\%$ & No & $\%$ & \\
\hline \multicolumn{8}{|l|}{ Feeding } \\
\hline Study group & 14 & 20 & 31 & 44.3 & 25 & 35.7 & \multirow{2}{*}{$\begin{array}{c}\mathrm{X} 2=37.5, \\
\mathrm{P}=0.000 \mathrm{HS}\end{array}$} \\
\hline Control & 40 & 57.1 & 30 & 42.9 & 0 & 0 & \\
\hline \multicolumn{7}{|l|}{ Having a shower } & \multirow{2}{*}{$\begin{array}{c}\mathrm{X} 2=64.8 \\
\mathrm{P}=0.000 \mathrm{HS}\end{array}$} \\
\hline $\begin{array}{l}\text { Study group } \\
\text { Control }\end{array}$ & $\begin{array}{l}12 \\
40\end{array}$ & $\begin{array}{l}17.1 \\
57.1\end{array}$ & $\begin{array}{l}14 \\
30\end{array}$ & $\begin{array}{c}20 \\
42.9\end{array}$ & $\begin{array}{c}44 \\
0\end{array}$ & $\begin{array}{c}62.9 \\
0\end{array}$ & \\
\hline \multicolumn{7}{|l|}{$\begin{array}{l}\text { Patient general } \\
\text { appearance }\end{array}$} & \multirow{2}{*}{$\begin{array}{l}\mathrm{X} 2=14.8 \\
\mathrm{P}=0.000 \mathrm{HS}\end{array}$} \\
\hline $\begin{array}{l}\text { Study group } \\
\text { Control }\end{array}$ & $\begin{array}{l}0 \\
0\end{array}$ & $\begin{array}{l}0 \\
0\end{array}$ & $\begin{array}{l}11 \\
32\end{array}$ & $\begin{array}{l}15.7 \\
45.7\end{array}$ & $\begin{array}{l}59 \\
38\end{array}$ & $\begin{array}{l}84.3 \\
54.3\end{array}$ & \\
\hline \multicolumn{7}{|l|}{ Patient' wearing } & \multirow{2}{*}{$\begin{array}{l}\mathrm{X} 2=74.4 \\
\mathrm{P}=0.000 \mathrm{HS}\end{array}$} \\
\hline $\begin{array}{l}\text { Study group } \\
\text { Control }\end{array}$ & $\begin{array}{c}0 \\
26\end{array}$ & $\begin{array}{c}0 \\
37.1\end{array}$ & $\begin{array}{c}6 \\
30 \\
\end{array}$ & $\begin{array}{c}8.6 \\
42.9 \\
\end{array}$ & $\begin{array}{l}64 \\
14 \\
\end{array}$ & $\begin{array}{l}91.4 \\
20\end{array}$ & \\
\hline $\begin{array}{l}\text { Patient defecation } \\
\text { Study group } \\
\text { Control }\end{array}$ & $\begin{array}{c}1 \\
18 \\
\end{array}$ & $\begin{array}{c}1.5 \\
25.7\end{array}$ & $\begin{array}{c}5 \\
38 \\
\end{array}$ & $\begin{array}{c}7.1 \\
54.3 \\
\end{array}$ & $\begin{array}{l}64 \\
14 \\
\end{array}$ & $\begin{array}{c}91.4 \\
20 \\
\end{array}$ & $\begin{array}{c}\mathrm{X} 2=72.6 \\
\mathrm{P}=0.000 \mathrm{HS}\end{array}$ \\
\hline \multicolumn{7}{|l|}{ Patient urination } & \multirow{2}{*}{$\begin{array}{l}X 2=45.6 \\
P=0.000 \mathrm{HS}\end{array}$} \\
\hline $\begin{array}{l}\text { Study group } \\
\text { Control }\end{array}$ & $\begin{array}{c}5 \\
14 \\
\end{array}$ & $\begin{array}{l}7.1 \\
20 \\
\end{array}$ & $\begin{array}{l}18 \\
48 \\
\end{array}$ & $\begin{array}{l}25.7 \\
68.6 \\
\end{array}$ & $\begin{array}{c}47 \\
8 \\
\end{array}$ & $\begin{array}{l}67.2 \\
11.4 \\
\end{array}$ & \\
\hline \multicolumn{8}{|l|}{ Patient use bathroom } \\
\hline $\begin{array}{l}\text { Study group } \\
\text { Control }\end{array}$ & $\begin{array}{l}1 \\
8\end{array}$ & $\begin{array}{c}1.4 \\
11.4 \\
\end{array}$ & $\begin{array}{c}7 \\
38\end{array}$ & $\begin{array}{c}10 \\
54.3\end{array}$ & $\begin{array}{l}62 \\
24 \\
\end{array}$ & $\begin{array}{l}88.6 \\
34.3 \\
\end{array}$ & \multirow[t]{2}{*}{\begin{tabular}{|l|}
$\mathrm{X} 2=43.5$, \\
$\mathrm{P}=0.000 \mathrm{HS}$
\end{tabular}} \\
\hline \multicolumn{7}{|l|}{$\begin{array}{l}\text { Patient movement } \\
\text { from bed to } \\
\text { wheelchair and back }\end{array}$} & \\
\hline $\begin{array}{l}\text { Study group } \\
\text { Control }\end{array}$ & $\begin{array}{c}0 \\
56\end{array}$ & $\begin{array}{c}0 \\
80\end{array}$ & $\begin{array}{c}9 \\
14\end{array}$ & $\begin{array}{c}12.9 \\
20\end{array}$ & $\begin{array}{c}61 \\
0\end{array}$ & $\begin{array}{c}87.1 \\
0\end{array}$ & \multirow[t]{2}{*}{$\begin{array}{c}\mathrm{X} 2=11.8 \\
\mathrm{P}=0.000 \mathrm{HS}\end{array}$} \\
\hline \multicolumn{7}{|l|}{$\begin{array}{l}\text { Stepping the } \\
\text { ladder(stairs) }\end{array}$} & \\
\hline $\begin{array}{l}\text { Study group } \\
\text { Control }\end{array}$ & $\begin{array}{c}2 \\
20\end{array}$ & $\begin{array}{c}2.8 \\
28.6\end{array}$ & $\begin{array}{l}13 \\
10\end{array}$ & $\begin{array}{l}16.6 \\
14.3\end{array}$ & $\begin{array}{l}55 \\
40\end{array}$ & $\begin{array}{l}78.6 \\
57.1\end{array}$ & $\begin{array}{c}\mathrm{X} 2=21.3, \\
\mathrm{P}=0.000 \mathrm{HS}\end{array}$ \\
\hline \multicolumn{7}{|l|}{$\begin{array}{l}\text { Movement at surface } \\
\text { level }\end{array}$} & \multirow[b]{2}{*}{$\begin{array}{r}\mathrm{LR}=118.2, \\
\mathrm{P}=0.000 \mathrm{HS}\end{array}$} \\
\hline $\begin{array}{l}\text { Study group } \\
\text { Control }\end{array}$ & $\begin{array}{l}0 \\
8\end{array}$ & $\begin{array}{c}0 \\
11.4\end{array}$ & $\begin{array}{l}15 \\
62\end{array}$ & $\begin{array}{l}21.4 \\
88.6\end{array}$ & $\begin{array}{c}55 \\
0\end{array}$ & $\begin{array}{c}78.6 \\
0\end{array}$ & \\
\hline
\end{tabular}

Vol. 18 No. 1 May, 2020 
Figure 2: Effect of home based nursing intervention on living of daily activities (LDAs) categories

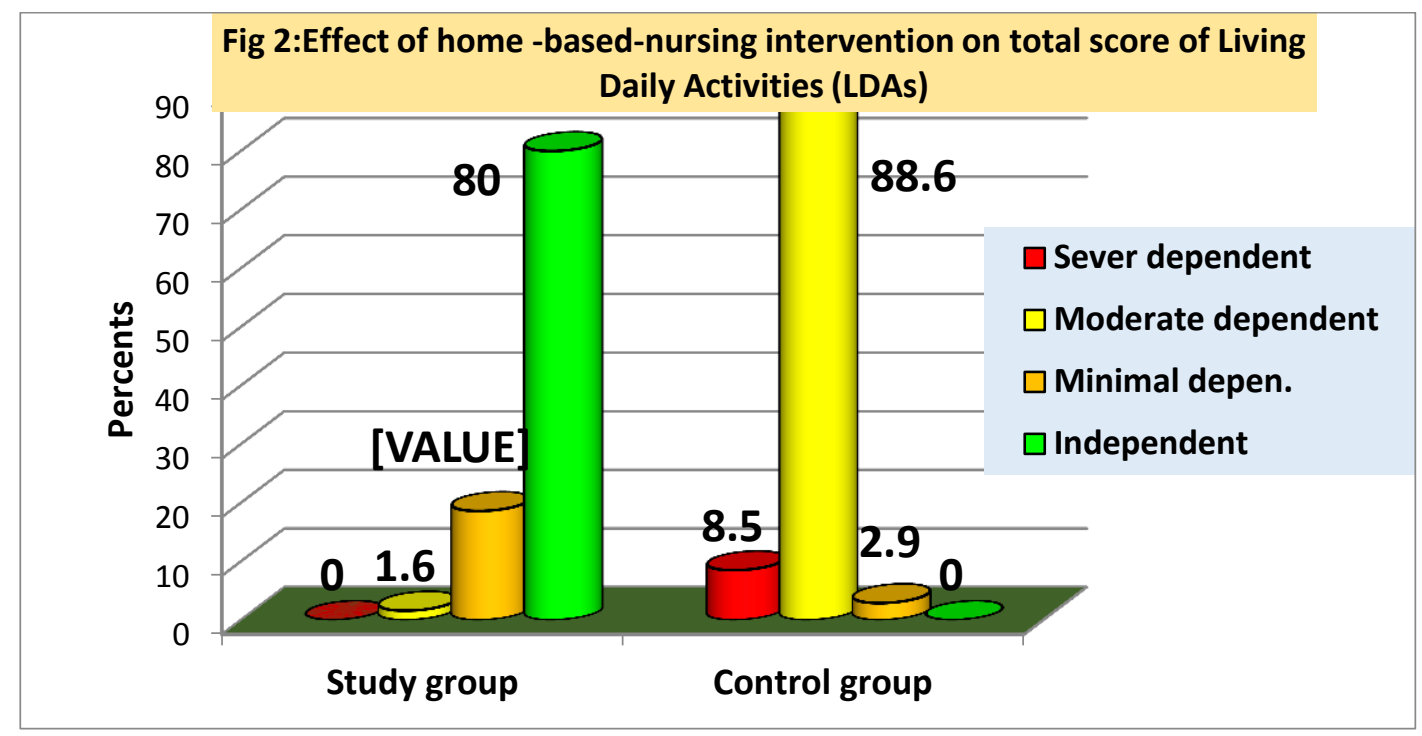

Table (5): Effect of home-based nursing intervention on pain after CABG among studied patient's groups $(\mathrm{N}=140)$

\begin{tabular}{|c|c|c|c|c|c|c|}
\hline \multirow{2}{*}{\multicolumn{2}{|c|}{ Visual Analog Scale }} & \multicolumn{3}{|c|}{ Groups } & \multirow{2}{*}{$\begin{array}{l}\text { Test of } \\
\text { Sig. }\end{array}$} & \multirow{2}{*}{ P-value } \\
\hline & & \multicolumn{2}{|c|}{$\begin{array}{l}\text { Study Gr. } \\
\text { N0. } \%\end{array}$} & $\begin{array}{l}\text { Control } \\
\text { N0. } \%\end{array}$ & & \\
\hline \multirow{3}{*}{$\begin{array}{l}\text { Analog } \\
\text { visual scale }\end{array}$} & $\begin{array}{l}0-3 \text { mild } \\
\text { pain }\end{array}$ & & 45.7 & $\begin{array}{ll}4 & 5.7\end{array}$ & \multirow{3}{*}{$\mathrm{X} 2=21.8$} & \multirow{3}{*}{$\mathrm{P}=0.000 \mathrm{HS}$} \\
\hline & $4-6$ moderate pain & 26 & 37.1 & $25 \quad 35.7$ & & \\
\hline & $\begin{array}{l}7-10 \text { severe } \\
\text { pain }\end{array}$ & & 17.2 & $41 \quad 58.6$ & & \\
\hline \multicolumn{2}{|l|}{ Mean \pm SD } & \multicolumn{2}{|c|}{$2.9 \pm 1.1$} & $8.3 \pm 1.7$ & $\mathrm{t}=14.2$ & $\mathrm{P}=0.000 \mathrm{HS}$ \\
\hline \multirow{3}{*}{\begin{tabular}{|l|} 
Description \\
of pain
\end{tabular}} & numbness & & 31.4 & $26 \quad 37.1$ & \multirow{3}{*}{$\mathrm{X} 2=2.6$} & \multirow{3}{*}{$\mathrm{P}=0.3 \mathrm{NS}$} \\
\hline & muscle strain & 20 & 28.6 & $12 \quad 17.1$ & & \\
\hline & burning & 28 & 40.0 & $32 \quad 45.7$ & & \\
\hline
\end{tabular}

\section{Cont. table 5}

Vol. 18 No. 1 May, 2020 
Tanta Scientific Nursing Journal

\begin{tabular}{|c|c|c|c|c|c|c|c|}
\hline \multirow{2}{*}{\multicolumn{2}{|c|}{ Visual Analog scale }} & \multicolumn{4}{|c|}{ Groups } & \multirow{3}{*}{ Test of sig. } & \multirow[b]{2}{*}{ P- value } \\
\hline & & \multicolumn{2}{|c|}{$\begin{array}{l}\text { Study Gr. } \\
\text { No. } \%\end{array}$} & \multicolumn{2}{|c|}{$\begin{array}{l}\text { Control } \\
\text { N0. } \%\end{array}$} & & \\
\hline \multirow[t]{4}{*}{ Site of pain } & in the chest & & 62.9 & & 25.7 & & \multirow{4}{*}{$\mathrm{P}=0.000 \mathrm{HS}$} \\
\hline & in the knee & 10 & 14.3 & 34 & 48.6 & \multirow{3}{*}{$\mathrm{LR}=28.5$} & \\
\hline & the back & 10 & 14.3 & & 22.9 & & \\
\hline & the shoulder & 6 & 8.6 & 2 & 2.9 & & \\
\hline \multirow{4}{*}{ Pain increase with } & Walk \& movement & 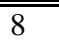 & 11.4 & 26 & 37.1 & \multirow{4}{*}{$\mathrm{X} 2=36.5$} & \multirow{4}{*}{$\mathrm{P}=0.000 \mathrm{HS}$} \\
\hline & Hard work & 48 & 68.6 & 14 & 20 & & \\
\hline & Using stairs & 12 & 17.1 & 18 & 25.7 & & \\
\hline & Others & 2 & 2.9 & 12 & 17.1 & & \\
\hline \multirow{4}{*}{ Pain decrease with } & Warm compresses & 26 & 37.1 & 22 & 31.4 & \multirow{4}{*}{$\mathrm{LR}=15.7$} & \multirow{4}{*}{$\mathrm{P}=0.001 \mathrm{Sig}$} \\
\hline & Period of rest & 12 & 17.1 & 32 & 45.7 & & \\
\hline & Simple exercise & 30 & 42.9 & 14 & 20 & & \\
\hline & Use analgesics & 2 & 2.9 & & 2.9 & & \\
\hline
\end{tabular}

\section{DISCUSSION}

Nurses playing a vital role in treatment as they are close to the patients and their 
families during all process of the disease.

Nurses can meet the rehabilitative care needs of patients through education, support, supervision and reinforcement. Regarding to previous study, nursing education in cardiac rehabilitation can improve health outcomes and reduce the risk of a new cardiac event. A health educational program organized by nurses for patients after a cardiac event or surgery improves patients' knowledge of their illness and awareness of behavioral changes to prevent a new event or readmission to hospital (Wang et al., 2012). ${ }^{(14) .}$

The aim of the current study was to determine the effect of home based nursing intervention on knowledge, daily living activity and pain on patient after coronary artery bypass graft surgery.

Regarding to age, the present study revealed that almost two thirds of the study group of patient with coronary artery bypass graft were between 31-50 years. This finding was congruent with the finding of Kerola, Kauppi, et al, (2012) ${ }^{(12) .}$ who studied. "How early in the course of rheumatoid arthritis does the excess cardiovascular risk appear?" ,.

Also, the rates are higher among men than women of a given age. This finding could be due to the level of health awareness and knowledge given to them that make them realize early the risk factors and performing continuous checkup and adopting healthy lifestyles.

According to the present study findings, patient knowledge about risk factors has improved significantly after implementing the home based nursing intervention which raised their awareness about high blood pressure, smoking, diabetes, lack of exercise, obesity, high blood cholesterol, poor diet, depression, and excessive alcohol as risk factors that can lead to CAD. This finding proved the first hypothesis which stated that patients who will receive the home based nursing intervention after coronary artery bypass graft will have improved knowledge score compared to control group ${ }^{(14)}$

In the present study, the majority of the study group were smokers and reported having many disease which are considered as risk factors for CAD. This finding was in agreement with Moran et al., (2014) ${ }^{(15)}$ who studied "The global burden of ischemic heart disease in 1990 and 2010: the Global Burden of Disease 2010 study", and reported that Coronary artery disease had a number of well determined risk factors. These include high blood 
pressure, smoking, diabetes, lack of exercise, obesity, high blood cholesterol, poor diet, depression, family history, and excessive alcohol. Also, Dai et al., (2016) (16). who studied and claimed that smoking and obesity were associated with about $36 \%$ and $20 \%$ of CAD cases. Smoking just one cigarette per day doubles the risk of CAD.

Regarding to psychological stress in the present study, most of the patients reported that it played an important role in causing the disease from their perception. This finding was in agreement with previous study conducted by Charlson, Moran, Freedman et al., (2013) (17). who studied" Genetics of coronary artery disease and myocardial infarction", and reported that stress appears to play a role in the occurrence of CAD cases.

Likewise, a study conducted by Kerola , (2012) ${ }^{(18)}$ who studied "How early in the course of rheumatoid arthritis does the excess cardiovascular risk appear?". and found that women who were free of stress from work, showed an increase in the diameter of their blood vessels, leading to decreased progression of atherosclerosis. In contrast, women who had high levels of work-related stress experienced a decrease in the diameter of their blood vessels and significantly increased disease progression. Also, people having type A behavior pattern - group of personality characteristics including time urgency, competitiveness, hostility, and impatience - is linked to an increased risk of coronary disease.

In addition, the study revealed that there was high statistical significance improvement in knowledge among study group than control groups about factors that lead to coronary artery disease after implementing the (HBNI), where about three fourth of the study group of patient with CABAG had good knowledge compared to about one third of the control group. This differences could be due to the success of the HBNI than the routine hospital rehabilitation program.

The level of knowledge among the study group on wound and self-care before attending the HBNP after coronary artery bypass grafting was unsatisfactory. However, the results showed high statistical significant improvement in self and wound care among the study group than the control group after attending the HBNI. This findings may contribute to the success of the HBNI where the study group were lacking the knowledge but once their awareness was raised they became more 
knowledgeable about the importance of self and wound care.

The current findings was supported by Graham etal., (2011) ${ }^{(11) .}$ who conducted a study in "Prevention of coronary artery disease" and found that up to $90 \%$ of cardiovascular disease may be preventable if established risk factors was avoided. Prevention involves adequate physical exercise, decreasing obesity, treating high blood pressure, decreasing cholesterol levels, and stopping smoking. Medications and exercise are roughly equally effective. High levels of physical activity reduce the risk of coronary artery disease by about $25 \%$. This identify a need for health education on postoperative self-care among the study group of patients with coronary artery bypass grafting. The health education on self-care after the coronary artery bypass grafting should be conducted by nurses on discharge and followed at home ${ }^{(12) .}$

Diet high in fruits and vegetables decreases the risk of cardiovascular diseases and death. Vegetarians have a lower risk of heart disease, possibly due to their greater consumption of fruits and vegetables. Evidence also suggests that the Mediterranean diet and a high fiber diet lower the risk of heart diseases.
On the other hand, Laz \& Berenson, (2015) (13) reported that although consulting a registered dietician is preferred regarding appropriate portion sizes and total calorie recommendations, improving nutrition knowledge may be beneficial regarding healthy and harmful foods. Community health nurse who conduct the discharge plan will provide a realistic ways to change bad eating habit. This means that a negative relationship exists between nutrition knowledge and physical activity, and body mass index (BMI), implying that those with lower knowledge scores will have higher BMI values. Furthermore, increases in knowledge regarding nutrition may promote healthy weight loss behavior. This finding supports the second hypothesis which stated that "Patients who will receive home based nursing intervention after coronary artery by bass will have better daily living activities score compared to control group", (14).

In the present study secondary prevention was preventing further squeal of already established disease. Lifestyle changes that have shown to be effective to this goal include :Weight control, smoking cessation, avoiding the consumption of trans-fats (in partially hydrogenated 
oils),decreasing psychosocial stress, exercise, like walking, jogging, or swimming, can reduce the risk of mortality from coronary artery disease ${ }^{(15,16) .}$. Aerobic exercise can help decrease blood pressure and the amount of blood cholesterol (LDL) over time. This finding is in agreement with the study conducted by Zhao, (2017) ${ }^{(17)}$. , about "Coronary Artery Bypass Grafting With and Without Manipulation of the Ascending Aorta which concluded that increased HDL cholesterol was considered as "good Cholesterol" reduces the risk of mortality from MI. In the present study, aerobic exercise after $\mathrm{CABG}$ will reduce the neither blood level of nor epinephrine which limits vasoconstriction of the arterioles and decreases the peripheral resistance to $\mathrm{BP}$, hence causing reductions in BP. Another possible reason for the reductions in SBP in this study could be that regular exercise also lowers HR due to activation of the parasympathetic division of the autonomic nervous system, which in turn allows the ventricles more time to fill with blood, ensuring adequate oxygen and nutrient delivery.

The present study revealed that cholesterol level in body was an important risk factor in occurrence of the disease. The findings showed that the majority of study group $(91.4 \%)$ reported high cholesterol level as a factor contributing to the disease. Likewise, previous study conducted by Seiler et al., (2013) (18). About Nano particles containing the pro-resolving peptide Ac2-26 protect against advanced atherosclerosis in hypercholesterolemic revealed that Cholesterol was an important factor involved in the pathogenesis of coronary artery disease. High levels of low-density lipoproteins (LDL) are implicated in coronary artery disease whereas high-density lipoproteins (HDL) are thought to have a protective role since they were involved in transportation of cholesterol away from the peripheral tissues.

Likewise, the finding of the study revealed that there was high statistical significance improvement among case than control patient about their knowledge related to prevention of post -operative complication after CABG. This finding supported the hypothesis that the "HBNI was effective in increasing patient knowledge and reduces the occurrence of complications and have aid positive recovery effect".

The effect of home based nursing intervention on pain : The American Occupational therapy Association for 
Study of Pain (2015) (19). defined pain as an undesirable sensory and emotional experience that is connected with actual or potential tissue damage, or characterized in terms of such damage. Pain can be categorized based on its duration into acute or chronic pain (WHO, 2012) ${ }^{(20)}$. Pain had also been reported to be one of the main sources of concern for cardiac surgery patients, and post-operative pain was still a significant clinical problem although there are major advances in pain management and treatment.

Pain syndromes that occur after cardiac surgery can be numerous and of visceral, musculoskeletal, or neurogenic origin. The findings of the current study showed that there was high statistical significance decrease on pain after CABG between the study and control group after implementing the home based nursing intervention.

The results of the current study illustrated that the reported level of pain after surgery decreased the quality of life for patients and affects their comfort level. This finding is congruent with the finding of Chou, (2016) (20). in Canada, who studied. Patient satisfaction with their pain management and comfort level after open heart surgery. and found that the incidence of chronic pain following cardiac surgery varies between $21 \%$ and $55 \%$. This finding confirm the third hypothesis which stated that "Patients who will receive home based nursing intervention after coronary artery by bass will have lower pain score compared to control group "The results of the current study revealed that there was high statistical significance improvement among case than control patient after CABG in the Barthel index scale (BIS) which measures activities of daily living after applying the home based nursing intervention. The results revealed that there was high statistical significance decrease on level, duration and type of pain after CABG between study and control groups which positively affect their quality of life. This finding is congruent with the reported findings of a study conducted by Murphy, (2015) $^{(21) .}$.

The VAS measurements have been included in the literature review as a method of describing patient experience of pain management, with low pain scores indicating more positive quality of life.

Activities of daily living: The current study illustrated that there was high statistical significance improvement among the study group than the control group after applying the home -based-nursing intervention on total score of Daily living Activities 
(DLAs). The finding of the present study was similar to that reported by Madssen et al., (2014) (17). who studied" Peak oxygen uptake after cardiac rehabilitation: a randomized controlled trial of a 12-month maintenance program versus usual care" and found that the mean scores of the variables were significantly higher in the intervention group following the intervention. This finding might contribute to the modifications of life style to a healthy one and reduced the dependency on others to care for him/her self.

Likewise, poor sleep quality was common among patients after coronary artery bypass graft surgery (CABG). Also, in a previous stud by Simeith et al., (2015) ${ }^{(18) .}$ about "Six-year follow-up of a randomized controlled trial examining hospital versus home-based exercise training after coronary artery bypass graft surgery", showed that psychological sleep management training, combining relaxation techniques, sleep hygiene, and cognitive techniques, could improve the SQ and in turn the total quality of life of patients.

These findings indicated that the quantity of exercise performed by the participants in the present study have been in line with the current recommended frequencies and intensities to reach and maintain optimal health. While the key objective of CR was to develop and improve QoL, postoperative pain levels and ADLs were significant indicators for evaluating the changes in physical and psychological well- being of cardiac patients.

Correspondingly, bodily pain, general health and vitality were also found to improve significantly. As a result of CABG surgery, some of the most important adverse consequences include forced inactivity, pain in the chest and vein harvest site and reduction of QoL, which may all contribute to severe stress and depression. Similarly, another study conducted on CABG patients by Nazari et al., (2012) (19). to assess the effect of rehabilitation on strength and balance, yielded significant results in the "chair stand and up-and-go tests". From the aforementioned study, it was evident that neuromotor training was imperative for increasing independence, confidence and reassurance.

These findings showed that the routine care provided by the routine cardiac rehabilitation clinic had slight positive impact on the control group compared to the effect of the HBNI. It included exercise training, life style modifications, 
medication adherence, and pain, sleep and dietary modifications improve the patient's quality of life and reduce the anxiety after CABG. According to the results of a study conducted by Jiang et al.(2013) (21). a nurse educational program can significantly improve the health behaviors and cardiac physiological risk parameters in coronary heart disease patients (Jiang and Wong, 2013) ${ }^{(22) .}$. Also, Safabakhsh et al,.( 2016) (18). who conducted that "The Effect of Health Promoting Programs on Patient's Life Style After Coronary Artery Bypass Graft-Hospitalized in Shiraz Hospitals." Conducted a study with 80 patients who underwent CABG surgery and reported that the education provided in the health-promotion program after the operation recommending positive changes in lifestyle reduce the risk factors of CAD and that the patients became more conscious about healthy behaviors. Observed that training and counseling for CAD patients increased the level of physical activity and diabetes compliance, patients must adapt to incorporating healthy lifestyle behaviors in areas such as nutrition habits, exercise, and social and work life (Safabakhsh , 2016) ${ }^{(18) .}$.The HBNI provided at discharge in the current study following open heart surgery was successful and increased the knowledge of patients, reduced pain, improved activities of daily living, sleep quality which in turn improve patients quality of life and was beneficial as it eliminates or reduces physical and emotional problems of the patient $^{(23-26) \text {. }}$

\section{Conclusion}

In the light of the present study, it can be concluded that:

The results of this study showed the effectiveness of the Home Based Nursing Intervention on improving patient knowledge, level of pain and quality of life. It indicated that such programs helped patients maintain a healthy lifestyle by controlling the risk factors. The results revealed that smoking, blood pressure control, frequency of physical activity, exercise, dietary behavior, activities of daily living and dependency level were modified in the study group with high statistical significant improvement in all aspect of the study. Improving cases awareness and the therapeutic lifestyle change effectively improve postoperative recovery and prognosis.

\section{RECOMMENDATION}

Based on the findings of the study, the following important recommendations are proposed: 
Regarding to the study finding nursing education in cardiac rehabilitation can improve health outcomes and reduce the risk of a new cardiac event. Also, due to the importance and effectiveness of postoperative rehabilitation interventions, the following are recommended:

1. It is a matter of great importance for nurses to meet the rehabilitative care needs of patients through education, support, supervision and reinforcement.

2. There is a need to include multidisciplinary team including surgeons, dietitian and nurse to take active part in such intervention.

3. The rehabilitation program has to start at the preoperative phase of patient care.

\section{References}

1. World Health Organization (WHO). Cardiovascular diseases, fact sheet \#317.Retrievedfrom

http://www.who.int/mediacentre/factsh eets/fs317/en/

2. -Collin C., Wade DT., Davies S. \& Horne V. "The Barthel ADL Index: a reliability study. Intl Disabil Stud.1988; 10(2): 61-63.

3. Bhimji S. Transmyocardial laser revascularization. Medscape. Retrieved from http://emedicine.medscape.com/article/ 428355-overview\#

4. McLaughlin MA. Cardiovascular care made incredibly easy (3rd ed.). Philadelphia: Wolters Kluwer. MedicineNet. Nitroglycerinsublingual, Nitrostat. Retrieved from http://www.medicinenet.com/nitroglyc erin-sublingual/article.htm

5. Heran BS. Exercise-based cardiac rehabilitation for coronary heart disease Cochrane Database SystRev. 2011;(7):CD001800

6. West RR, Jones, et al ,. Rehabilitation after myocardial infarction trial (RAMIT): multi- center and randamised controlled trial of comprehensive cardiac rehabilitation in patients following acute myocardial infarction. Heart. 2012;98:637-644.

7. Wang W, Chair SY, Thompson DR, Twinn SF. Health care professionals' perceptions of hospital-based cardiac rehabilitation in mainland China: an exploratory study. J Clin Nurs. ;2012 .18(24):3401-8. (1):94-101.

8. American Heart Association (AHA).. Heart disease and stroke statistics - at a glance. Retrieved from http://www.heart.org/idc/groups/ahama 
hpublic/@wcm/@sop/@smd/document s/downloadable/ucm_470704.

9. American Occupational Therapy Association (AOTA).. The role of occupational therapy in chronic disease management. Retrieved from http://www.aota.org/media/Corporate/F iles/AboutOT/Professionals/WhatIsOT HW/Facts/FactSheet_ChronicDisease Management.pdf

10. Charlson, FJ; Moran, AE; Freedman, G. Et al., "The contribution of major depression to the global burden of ischemic heart disease: a comparative risk assessment". BMC Medicine. (2013). 11: 250. Doi:10.1186/ 17417015-11-250

\section{Graham IM, Fallon N, Ingram S, et}

al. Rehabilitation of the patient with coronary heart disease. In V Fuster, RA Walsh, \& RA Harrington (Eds.), Hurst's the heart (13th ed.). New York: McGraw-Hill.2011

\section{Kerola, AM; Kauppi, MJ; Kerola, T;}

Nieminen, $\mathbf{T}$. "How early in the course of rheumatoid arthritis does the excess cardiovascular risk appear?". Annals of the Rheumatic Diseases. 2012,71 (10): $\quad$ 1606-
15. Doi:10.1136/annrheumdis-2012201334. PMID 22736093).

13. Laz T. . Berenson level of nutrition knowledge and its association with weight loss behaviours among lowincome reproductive-age women Journal of Community Health, 40 (3) (2015), pp. 542-548.

14. World Health Organization .Global Health Risks: Mortality and burden of disease attributable to selected major risks, World Health Organization, Geneva (2009). Retrived://www.who.int/healthinfo/glo bal_burden_disease/GlobalHealthRisks _report_full.pdf

15. Moran, AE; Forouzanfar, MH; Roth, GA. Et al.,"The global burden of ischemic heart disease in 1990 and 2010: the Global Burden of Disease 2010study".

Circulation.

$129(141493501$.

Doi:10.1161/

circulationaha.113.004046. PMC 4181 601).

16. Dai, Xuming, et al. "Genetics of coronary artery disease and myocardial infarction". World Journal of Cardiology. National Center for 
Biotechnology Information, U.S. National Library of Medicine. 2016 ,8 (1): 1-23. Doi:10.4330/wjc.v8.i1. 1. PMC 4728103.

\section{Madssen E, Arbo I, Granoien I, et al.}

Peak oxygen uptake after cardiac rehabilitation: a randomized controlled trial of a 12-month maintenance program versus usual care. Plos ONE 2014; 9: e107924.

18. Safabakhsh L, Arbabisarjou A, Jahantigh M, Nazemzadeh $M$, Rigi SN, Nosratzehi $\mathbf{S}$.The Effect of Health Promoting Programs on Patient's Life Style After Coronary Artery Bypass Graft-Hospitalized in Shiraz Hospitals. Glob J Health Sci $2016 ; 8: 154-9$.

\section{Menezes AR, Lavie CJ, Forman DE,} et al. Cardiac rehabilitation in the elderly. Progress in Cardiovascular Diseases, 2014 .57, 152-9.

20. Kuru N, Piyal B.. Gulhane Military Medical Academy Training Hospital, the Applicant Determination of Healthy Lifestyle Behaviors in Individuals Diagnosed Coronary Artery Disease. TAF Prev Med Bull; 2012,11:287-98.

21. Kurçer MA, Özbay A. Effects of patient education and counseling about life style on quality of life in patients with coronary artery disease. Anatol J Cardiol $2011 ; 1: 107-13$.

\section{Nazari N, Javaheri SAAH, Lamir} AR, et al.. Effect of cardiac rehabilitation on strength and balance in patients after coronary artery bypass graft. Zahedan Journal of Research in Medical Sciences; 2012 ;1(15):29-33.

23. Jiang X, Sit JW, Wong TK. A nurseled cardiac rehabilitation programme improves health behaviours and cardiac physiological risk parameters: evidence from Chengdu, China. J Clin Nurs. 2013 ;16(10):1886-97.

\section{Smith KM, mckelvie RS, Thorpe} KE, et al.. Six-year follow-up of a randomised controlled trial examining hospital versus home-based exercise training after coronary artery bypass graft surgery. Heart ;2011, 97: 11691174.

\section{Seiler C, Stoller E, Pitt B, \& Meier P.}

The human coronary collateral circulation: development and clinical importance. European Heart Journal, 2013.34(34), 2674-82.

26. Kerola, AM; Kauppi, MJ; Kerola, T; Nieminen, TV . "How early in the course of rheumatoid arthritis does the excess cardiovascular risk 


\section{Tanta Scientific Nursing Journal}

appear?". Annals of the Rheumatic

Diseases. 2012,71 (10): 1606-

15. Doi:10.1136/annrheumdis-2012-

201334. PMID 22736093).

27. Wewers M.E. and Lowe N.K. A

critical review of visual analogue

scales in the Measurement of clinical

phenomena. Research in Nursing and

Health, 13, 227-236. 1990.

Vol. 18 No. 1 May, 2020 